\title{
A saúde humana e a Amazônia no século XXI: reflexões sobre os objetivos do milênio
}

Hilton P. Silva - Professor, Coordenador do Setor de Antropologia Biológica e Chefe do Departamento de Antropologia do Museu Nacional, Universidade Federal do Rio de Janeiro (UFRJ)

\section{Resumo}

A Amazônia, além de um vasto e complexo ecossistema, é também o habitat de um considerável contingente populacional humano. Embora grande parte desse contingente esteja concentrada nas áreas urbanas, milhões de pessoas vivem nas áreas rurais. Essas populações incluem os diversos grupos indígenas, as populações caboclas, os quilombolas e os imigrantes recentes da várias partes do país. Pouco ainda se conhece sobre esses grupos, e menos ainda sobre sua situação de saúde e saneamento ambiental. Este artigo faz uma síntese dos principais desafios para a saúde das populações amazônicas e apresenta exemplos da precária situação de alguns grupos rurais. Observa-se que nesta região não será possível atingir os Objetivos do Milênio dentro dos prazos estabelecidos, e apresentam-se algumas propostas de políticas públicas na área de saúde e meio ambiente que, caso venham a ser implementadas, poderão, no futuro, melhorar as condições de saúde das populações amazônicas e, simultaneamente, promover a proteção do meio ambiente.

\section{Palavras-chave}

Meio ambiente, Brasil, saúde, caboclos, Pará.

\begin{abstract}
The Amazon region is known for its vastness and complex ecosystem. Nevertheless, this area is also the habitat of a considerable human contingent. Even though a large part of this population is concentrated in the urban areas, millions of people also live in the rural areas. These populations include several indigenous groups, traditional populations such as the caboclos and the quilombolas, and recent immigrants from several parts of Brazil. However, little is still known about these groups and even less about their health and environmental sanitation situation. This article provides an overview of some of the major challenges to the health of the Amazon populations and presents examples of the precarious situation of some rural groups. It is shown that in this region it will not be possible to reach the Millenium Objectives in the currently proposed timeframe, and some proposals for public policies in the areas of health and the environment are presented which, if implemented, will improve the health of the Amazonian populations and help to simultaneously promote environmental protection.
\end{abstract}

\section{Keywords}

Environment, Brazil, health, mestizo, Pará. 
Hilton P. Silva

\section{INTRODUÇÃO}

Discutir perspectivas sobre as complexas relações entre meio ambiente e saúde na Amazônia é um enorme desafio que, porém, jamais deixa de ser fascinante e necessário. Quando se trata da Amazônia, nada é simples como pode parecer à primeira vista, por isso, serão abordados neste artigo apenas alguns aspectos considerados fundamentais para a reflexão sobre a área da saúde, se queremos ter no horizonte futuro da Amazônia um desenvolvimento socioeconômico com bases minimamente sustentáveis.

Qualquer viajante na Amazônia percebe facilmente as grandes disparidades em termos de acesso aos serviços básicos de infraestrutura, como saneamento ambiental, atendimento de saúde, e serviços de tratamento de água, entre as áreas urbanas e as áreas rurais da região. Infelizmente, essa realidade mudou pouco e muito lentamente nas últimas décadas (COUTO et al., 2002; JATENE et al., 1993). Garantir o acesso a tais serviços para a maioria da população continua a ser um dos maiores desafios das políticas públicas para esta parte do Brasil (SILVA, 2004a).

Complexidade e diversidade são as características fundamentais da região amazônica. A Amazônia legal brasileira é formada por nove Estados, ocupa 61\% do território nacional e tem 12.5\% do contingente populacional do país. São mais de cinco milhões de quilômetros quadrados de terras e águas. Na Amazônia encontra-se a maior biodiversidade da terra, quase um terço da variabilidade genética estimada do planeta, a maior sociodiversidade da humanidade, as principais reservas estratégicas superficiais de água doce e algumas das maiores, e ainda intocadas, reservas de minerais estratégicos do mundo.

Quantos países cabem na Amazônia? E quantas etnias e culturas? Com alguma licença poética, na Amazônia cabe quase o mundo inteiro. O problema é que, se muito se conhece sobre outros países e culturas do resto do mundo, muito pouco ainda se sabe sobre esse mundo que é a Amazônia.

Por exemplo, há muito se fala, escreve, filma, fotografa e documenta de várias formas a enorme biodiversidade e a importância ecológica da

\footnotetext{
${ }^{1}$ O Ministério das Cidades tem recomendado o uso da expressão "saneamento ambiental" em vez de "saneamento básico".
} 
Amazônia para o planeta; no entanto, nessas expressões todas, destacase a pouca importância dada ao homem e à mulher amazônicos. E, quando eles aparecem, são tratados como parte da paisagem natural, ou caracterizados como agressores eternos do meio ambiente. Ambas as formas de representação são incorretas e perpetuam mitos como o do "bom selvagem" ou o do conflito insolúvel entre o ser humano e a natureza. Freqüentemente, quando se trata de populações nãoameríndias, a mensagem, equivocada, é a de que determinados setores, como os imigrantes, o campesinato rural, ou determinados movimentos sociais são eternamente nocivos ao meio ambiente; omite-se, ou ignorase, o fato de que essas populações são, também, detentoras e guardiãs de vasto conhecimento ecológico, advindo de séculos de vivência na região (CUNHA; ALMEIDA, 2002; DIEGUES, 2000; LISBOA, 2002; MORÁN, 1981, 1983).

A bioantropologia encarregou-se, há décadas, de demonstrar a falácia que é tentar separar o ser humano do seu meio ambiente ecológico (BAKER; LITTLE, 1976; KORMONDY; BROWN, 2001). Nos últimos milhões de anos, os seres humanos e seus antepassados contribuíram de forma decisiva para o surgimento e a manutenção de muitos dos ecossistemas naturais existentes hoje, inclusive a Amazônia (BENCHIMOL, 1998; DENEVAN, 2002; HECKENBERGER et al., 2003). Portanto, desconsiderar os seres humanos em qualquer aspecto da discussão ambiental é uma grande insensatez. Felizmente, há setores da sociedade, entre os quais algumas organizações não-governamentais (ONGs) - ainda que poucas - e áreas do governo federal, dos governos estaduais e municipais que já apresentam uma perspectiva mais avançada sobre a relação entre os seres humanos e o meio ambiente. Esse movimento está cada vez mais forte no Brasil (ALIER, 1998; FATHEUER et al., 1998). No entanto, dadas dificuldades enfrentadas pela maioria dos amazônidas, os desafios ainda são enormes.

\section{OS PRINCIPAIS DESAFIOS PARA A SAÚDE NA AMAZÔNIA A transição socioecológica e a carga dupla de doenças}

Enquanto já se nota alguma predisposição da comunidade acadêmica e da mídia para incluir os seres humanos na equação ecológica, faltam ainda dados que permitam essa inclusão de forma correta com seus efeitos calibrados e concretamente avaliados. Embora a Amazônia Legal tenha o maior contingente populacional rural do país, pouco se sabe sobre a diversidade sociocultural dessas populações, e 
Hilton P. Silva

muito menos é conhecido sobre sua situação de saúde. Os principais esforços de pesquisas têm se concentrado em duas grandes vertentes: as capitais e outras áreas urbanas, como Belém, Santarém, Manaus, Boa Vista e Rio Branco, e as populações indígenas. Nessas duas frentes, muito embora o quadro esteja ainda incompleto, já há um acúmulo considerável de informações sobre saúde e condições de vida das populações, como pode ser visto nos dados do DATASUS, do Ministério da Saúde (BRASIL, 2005) e em publicações nacionais e internacionais (COIMBRA JR. et al., 2003; COUTO et al., 2002; JATENE et al., 1993).

No entanto, há um outro segmento da população amazônica sobre o qual conhecemos menos ainda: o campesinato (as populações rurais não-indígenas, caboclos e ribeirinhos). Esse segmento, que o antropólogo britânico Stephen Nugent chama de população "invisível" (NUGENT, 1993), permanece no limbo científico (SILVA, 2001). Como resultado, um percentual expressivo da população da região é simplesmente ignorado pelas estatísticas oficiais. Como ocorria há cem ou duzentos anos, milhares de pessoas continuam a nascer e a morrer nos rincões amazônicos todos os anos sem que o poder público tome conhecimento de sua existência. Além disso, enquanto a era da tecnologia leva o telefone celular e a televisão a quase todos os cantos da região, a Amazônia continua a apresentar o segundo mais alto índice do país de mortes por "causas mal definidas", isto é, não identificadas pelo médico que assinou o atestado de óbito (CFM, 2005; JATENE et al., 1993), perdendo apenas para alguns dos estados do Nordeste. Isto indica que essas pessoas morrem antes de conseguir acesso a qualquer tipo de assistência médica.

Em geral, apesar de alguns avanços nas últimas décadas, os dados disponíveis sobre a saúde no Brasil ainda deixam muito a desejar. Porém, as informações existentes compõem, para a Amazônia em particular, um quadro bastante preocupante. Embora muitos dos indicadores epidemiológicos e sociodemográficos tradicionais (mortalidade infantil e materna, expectativa de vida ao nascer, cobertura vacinal da população, longevidade, status nutricional, acesso à educação básica etc.) tenham apresentado no país melhoras nos últimos vinte anos (IBGE, 2004; OPAS, 2002), na Amazônia, os avanços, quando ocorrem, têm sido bem mais lentos e irregularmente distribuídos, privilegiando, invariavelmente, os principais centros urbanos da região, em detrimento do grande contingente populacional das zonas rurais (BRASIL, 2004a; COSTA et al., 1993; FERREIRA et al., 2000; OPAS, 2002; SILVA, 2004a, 2004b).

Dados dos últimos documentos do Observatório da Cidadania do Pará (FAOR, 2003a) mostram que, na maior bacia hidrográfica do planeta, 
apenas $42 \%$ dos domicílios (e apenas em áreas urbanas) têm abastecimento regular de água. Essa informação ganha relevância quando confrontada com os dados da Organização Mundial da Saúde (OMS), que demonstram que as doenças de veiculação hídrica são responsáveis por 65\% das internações hospitalares de crianças até 10 anos de idade e por $80 \%$ das enfermidades que ocorrem nos países em desenvolvimento (RIBEIRO; MARIN, 2002; WHO, 2000). Para usar um exemplo paraense, o trabalho de Silva $(2001,2003,2006)$ em populações ribeirinhas do médio rio Amazonas mostra a relação existente entre fonte de água para consumo doméstico e prevalência de múltiplas parasitoses intestinais (ver Tabela 1). Estudos em outras populações da Amazônia têm demonstrado, também, níveis superiores a 20\% de poliparasitismo intestinal (DIAS et al., 1982; FERRARONI et al., 1979; IEC, 2000), o que reforça a relação entre acesso a saneamento ambiental e incidência de parasitoses intestinais.

Tabela 1: Percentagem de indivíduos infectados com parasitas intestinais em três populações ribeirinhas.

\begin{tabular}{|c|c|c|c|}
\hline Parasitas & $\begin{array}{c}\text { Caxiuanã } \\
\% \text { da população }\end{array}$ & $\begin{array}{c}\text { Aracampina } \\
\% \text { da população }\end{array}$ & $\begin{array}{c}\text { Santana } \\
\% \text { da população }\end{array}$ \\
\hline Ascaris lumbricoides & 57.8 & 13.8 & 6.1 \\
\hline Strongyloides stercoralis & 5.2 & 0.0 & 0.6 \\
\hline Tricocephalus trichiurus & 18.1 & 0.0 & 0.0 \\
\hline Ancylostoma duodenale & 19.8 & 6.5 & 5.2 \\
\hline Enterobius vermicularis & 0.0 & 0.8 & 0.2 \\
\hline Escherichia coli & 16.4 & 1.1 & 0.0 \\
\hline Entamoeba histolytica & 31.0 & 30.1 & 48.7 \\
\hline Giardia lamblia & 5.2 & 39.8 & 25.4 \\
\hline Endolimax nana & 37.1 & 0.0 & 13.1 \\
\hline Blastocistis hominis & 20.4 & 21.1 & 6.7 \\
\hline Múltiplos parasitas & 43.0 & 37.0 & 20.8 \\
\hline
\end{tabular}

Fonte: SILVA (2001, 2006).

Nas três populações apresentadas na Tabela 1 não há saneamento ambiental ou acesso à água encanada, e as famílias dependem dos rios e córregos, como fonte de água para todos os usos domésticos (SILVA, 2001). Como descrito naquele trabalho, embora as três populações sejam rurais e não tenham acesso a saneamento ambiental, as duas populações em situação socioecológica mais vulnerável Caxiuanã e Aracampina - apresentam, em média, maior prevalência de parasitoses intestinais. 
Hilton P. Silva

O Observatório da Cidadania do Pará (FAOR, 2003a) aponta, ainda, que apenas 6,5\% dos domicílios no Pará, aproximadamente, estão ligados a redes de esgotos. O quadro das indústrias, provavelmente, não é em nada melhor. Na região norte como um todo, menos de $20 \%$ dos domicílios estão conectados a redes de esgotos. Um provável efeito disso é que, de acordo com estimativas recentes, grande parte das fontes de captação de água para consumo humano está contaminada com poluentes de diversos tipos, de fezes humanas a metais pesados (COUTO et al., 2002; LISBOA, 2002).

Em termos de Índice de Desenvolvimento Humano (IDH), taxas de analfabetismo, proporção de pobres na população, mortalidade infantil, mortalidade perinatal e mortalidade materna, os estados da Amazônia apresentam dados melhores apenas que os dos estados mais pobres do Nordeste (BRASIL, 2004a; COUTO et al., 2002; FAOR, 2003b; UFRGS; UFPA; PUC-Minas; PNUD; IDHS, 2004). Tomando-se como exemplo o Estado do Pará, de acordo com o Mapa da Fome (FGV, 2001), o IBGE estima que $41 \%$ dos cerca de 6 milhões de habitantes do Estado são indigentes. Entre a área urbana e a área rural, são cerca de 2,5 milhões de pessoas nessa situação. Entre os principais pólos de pobreza estão Ananindeua, Marituba e Santa Bárbara, além da periferia de Belém, a cidade com a maior proporção de favelados do Brasil segundo o IBGE. O Pará é recordista em utilização de trabalho escravo no Brasil; quase 2000 pessoas foram encontradas pelas autoridades nessa situação em 2002 (FAOR, 2003a). Os dados sobre a violência no campo, o trabalho e a situação infantil são igualmente alarmantes. Outro dado grave é que, de acordo com dados do SUS (BRASIL, 2004b), tanto na cidade quanto no campo é elevado o índice de gravidez na adolescência, fato que está diretamente ligado à falta de acesso a informações básicas em saúde, à baixa escolaridade das meninas e dos meninos, à ausência de opções de lazer e trabalho e à falta de redes de suporte institucional.

De acordo com dados do Fundo das Nações Unidas para a Infância (UNICEF) e do Programa das Nações Unidas para o Desenvolvimento (PNUD), ainda é muito elevado também o índice de desnutrição infantil em toda a região. Os trabalhos de Silva (2001, 2002a) e do Programa Pobreza e Meio Ambiente (POEMA) da Universidade Federal do Pará (UFPA) (relatório não publicado) com algumas populações ribeirinhas exemplificam isso. Conforme demonstrado na Tabela 2, que compara dados de três populações que vivem em diferentes áreas geográficas do Pará, em média, até 50\% das crianças investigadas apresentam algum grau de desnutrição quando comparadas com os dados de referência internacionais. 
Tabela 2: Situação de três populações caboclas do Pará em relação à subnutrição, ao atraso agudo e ao atraso crônico de crescimento.

\begin{tabular}{l|l|l|c|c}
\hline População & Grupo etário & Subnutrição \% & $\begin{array}{c}\text { Atraso } \\
\text { agudo de } \\
\text { crescimento \% }\end{array}$ & $\begin{array}{c}\text { Atraso } \\
\text { crônico de } \\
\text { crescimento \% }\end{array}$ \\
\hline Caxiuanã $^{*}$ & $0-10$ & 57,2 & 34,8 & 79,6 \\
\hline Ituqui* $^{\text {Praia Grande** }}$ & $0-10$ & 49,3 & 26,6 & 72,1 \\
\hline
\end{tabular}

Fontes: *SILVA (2001); **dados não publicados cedidos pelo POEMA/UFPA (1994).

Do ponto de vista de outras minorias vulneráveis, uma situação crítica é a dos remanescentes dos vários quilombos espalhados pela Amazônia. Estimativas de organizações não-governamentais apontam que em até 80\% deles não há infra-estrutura de saneamento ambiental, unidades de saúde ou escolas oficiais. O mesmo pode ser dito sobre centenas de vilas e povoados ribeirinhos da região.

As populações não-indígenas e indígenas que vivem perto ou dentro de áreas protegidas (florestas nacionais, parques nacionais, áreas de proteção ambiental e outras unidades definidas no Sistema Nacional de Unidades de Conservação (SNUC)) também se encontram geralmente em situação crítica, pois legalmente não podem explorar os seus recursos naturais tradicionais e, por seu isolamento, tampouco têm acesso aos mercados e aos bens e serviços oferecidos pelo Estado em outras áreas. A população da Floresta Nacional de Caxiuanã, por exemplo (Tabelas 1 e 2), tem os piores indicadores em relação à desnutrição e prevalência de parasitoses intestinais, além de cáries, dermatites, diarréias e infecções respiratórias (SILVA, 2001, 2002a, 2003, 2006).

Em relação às doenças infecciosas mais graves, em toda a Amazônia, a malária, as hepatites virais, a hanseníase, a tuberculose, a leishmaniose, a dengue e mais recentemente a AIDS continuam a ser grandes desafios para a saúde pública. Há na região uma incidência de malária maior que a de muitos países da África subsaariana; a hanseníase, a dengue, a leishmaniose e a tuberculose ainda são endemias prevalentes, e pouco se sabe sobre a situação da sorologia das hepatites virais e do HIV, especialmente entre populações rurais (SILVA, 2002b, 2004b). No caso da malária, em muitas regiões, seus números continuam a crescer, sendo as cepas multirresistentes de Plasmodium falciparum cada vez mais freqüentes (UNEP, 2003). No caso particular da infecção pelo HIV, a tendência nacional aponta para a feminização, a pauperização e a interiorização cada vez maiores da epidemia, que afeta indivíduos 
Hilton P. Silva

nos segmentos adultos mais jovens da população, principalmente na faixa dos 15 aos 25 anos de idade (BRASIL, 2004b; BRITO et al., 2000; GUIMARÃES, 2001; SILVA, 2002b). Com a confirmação dessa tendência, os efeitos da infecção pelo HIV serão catastróficos nas populações rurais, já tão sofridas e desassistidas em termos de saúde.

Por outro lado, estudos têm demonstrado o surgimento do que a OMS chama de double burden (ou carga dupla): o quadro epidemiológico apresenta ainda altas taxas de doenças infecciosas, mas começam a emergir, em números cada vez maiores, as doenças crônico-degenerativas, como a hipertensão arterial, o diabetes, a obesidade e o câncer, que já estão entre as principais causas de morte nos estados da Amazônia (BRASIL, 2004a; SILVA 2004a). Por exemplo, comparando os níveis de pressão arterial e a prevalência de doença hipertensiva nas três populações ribeirinhas do médio rio Amazonas apresentadas nas tabelas anteriores (Caxiuanã, Aracampina e Santana), pode-se ter uma idéia da dinâmica da carga dupla nas populações amazônicas.

Nos três grupos investigados, além da alta prevalência de parasitoses intestinais, doenças respiratórias agudas, cáries, dermatites e outras doenças infecciosas agudas (as três áreas são relativamente livres de malária) (SILVA, 2001), começa a haver, também, um aumento nos níveis de pressão arterial, especialmente entre as mulheres. Cerca de $20 \%$ dos homens e de $25 \%$ das mulheres nas três comunidades apresentam níveis pressóricos elevados (PA ${ }^{3}$ 140/90 mmHg em duas medições consecutivas em períodos diferentes (STEIN, 1994)). Aproximadamente 60\% dos hipertensos são mulheres, e 28\% são pessoas com menos de 45 anos de idade (SILVA et al., 2006). Apenas uma pessoa estava em tratamento com anti-hipertensivos na época do estudo.

Em relação à obesidade (Índice de Massa Corporal 3 30), a prevalência entre os homens é de 18.9\%, 22.9\% e 20.8\% em Caxiuanã, Aracampina e Santana respectivamente. Mulheres obesas representam 11.1\%, 38.6\%, e 44.6\% respectivamente, nos três grupos (SILVA, 2001).

Apesar da amostra quantitativamente limitada, os índices de hipertensão e obesidade nesses três grupos são maiores que os de outras populações rurais já investigadas na região (SILVA et al., 1995; SILVA; ECKHARDT, 1994) e similares aos de populações de grandes áreas urbanas do Brasil e do mundo (DRESSLER; SANTOS, 2000; FEIJÃO et al., 2005; MONTEIRO et al., 2000; YUSUF et al., 2001), o que deve fazer soar um alerta para os potenciais custos humanos, sociais e econômicos acarretados pela carência de políticas públicas de saúde solidamente 
embasadas em dados de campo regionalizados, que considerem a complexidade das mudanças socioecológicas em curso na Amazônia.

Além da dificuldade de obtenção de informações confiáveis sobre as áreas rurais, em geral, a qualidade dos dados em saúde disponíveis no Brasil é um outro grave problema "epidemiológico" a resolver. Por exemplo: em 1997, o Ministério da Saúde estimava a mortalidade infantil no país em 16,6/1000 nascimentos, enquanto o IBGE a estimava em 36,2/1000, mais que o dobro. Ainda hoje, cerca de 25\% dos óbitos registrados (quase 40\% no Maranhão) resultam de "causas mal definidas" ou não diagnosticadas (CFM, 2005). Como dito anteriormente, na Amazônia rural, milhares ainda nascem e morrem sem qualquer atestado da sua existência. Essa situação não mudou em várias décadas (JATENE et al., 1993) e é, provavelmente, a maior demonstração da falta de Cidadania que atinge a maioria dos amazônidas.

\section{A QUESTÃO DA CARÊNCIA DE PROFISSIONAIS}

Uma outra questão fundamental a ser resolvida é a carência de profissionais de saúde na região. Os dados disponíveis (DUARTE et al., 2002; BRASIL, 2004a;) demonstram que há uma grande concentração de recursos materiais e de profissionais da saúde nas capitais e nas maiores cidades dos estados do Norte, e carências em outras áreas, havendo ainda municípios onde, mesmo na área urbana, a assistência básica à saúde é extremamente precária ou inexistente (FAOR, 2003a). Essa má distribuição de recursos humanos, e também materiais, avilta a situação dos profissionais da região. Em função da competição, são obrigados a trabalhar por baixos salários e em condições freqüentemente precárias nos principais centros urbanos - aliás, uma situação que se repete nas grandes cidades em todo o Brasil. Já nas áreas rurais, as prefeituras têm de oferecer salários altíssimos (e muitas vezes inviáveis de manter a médio prazo) para tentar fixar os profissionais nas menores localidades, onde as condições infra-estruturais, como acesso a transporte, métodos diagnósticos, leitos para internação, salas para cirurgia, e insumos para tratamento são, via de regra, insuficientes, o que dificulta sobremaneira a atuação dos poucos profissionais que se dispõem a trabalhar nesses locais.

Além disso, os dados mais recentes disponíveis no Conselho Federal de Medicina (CFM, 1998) demonstram que o número de profissionais em diversas especialidades na região é insuficiente para atender as demandas. Essa situação gerou o aparecimento, no mercado, especialmente no interior, de médicos provenientes de outros países da 
Hilton P. Silva

América do Sul, muitos com formação de qualidade duvidosa, o que coloca em risco a população atendida.

Por outro lado, atualmente, a formação curricular dos profissionais da saúde, baseada grandemente na dependência de equipamentos de alta tecnologia para diagnósticos, custosos equipamentos e insumos para tratamento (ARRUDA, 2001), dificulta a captação e a retenção dos profissionais na área rural e nas pequenas cidades do interior, onde esses recursos são escassos. Por isso, além de mudanças da infraestrutura e das políticas de saúde ora vigentes, um aspecto fundamental para a mudança das condições de saúde das populações amazônicas é o planejamento e a implementação de uma ampla reforma nos currículos da área de saúde da região. É necessária uma real transformação conceitual e prática, visando formar profissionais que compreendam a realidade amazônica, capazes de atuar de maneira segura e eficaz dentro dessa realidade, engajados, e que não tenham receio de encarar os desafios dos politraumatizados urbanos, nem de conduzir com confiança as equipes do Programa Saúde da Família (PSF) nos rincões regionais.

\section{CONSIDERAÇÕES FINAIS}

Em abril de 2005, foram lançadas no Brasil as dezoito "Metas do Milênio" da Organização das Nações Unidas (ONU) e os oito "Objetivos do Milênio", do PNUD. Os objetivos são: erradicação da pobreza extrema e da fome; universalização do acesso à educação primária; promoção da igualdade entre os gêneros; redução da mortalidade infantil; melhoria da saúde materna; combate a AIDS, malária e outras doenças; promoção da sustentabilidade ambiental; organização de parcerias para o desenvolvimento (http://www.objetivosdomilenio.org.br/ , acesso em 6/ 6/06). As Metas da ONU e os Objetivos do PNUD superpõem-se consideravelmente, uma vez que ambas as propostas fazem parte de uma agenda socioeconômico-sanitário-ambiental ampla, cujas raízes estão, por exemplo, na Conferência de Estocolmo, em 1972, na Agenda 21, que é o principal documento resultante da Eco-92 no Rio de Janeiro (BRASIL, 2001), e na Agenda do Milênio da ONU. Um conjunto de documentos preparado por especialistas de várias instituições brasileiras para o PNUD apresenta detalhadamente o quadro nacional para cada um dos objetivos e suas projeções (UFRGS; UFPA; PUC-Minas; PNUD; IDHS, 2004). Em geral, o Brasil está bastante aquém do cumprimento da maioria das metas e objetivos, sendo a situação particularmente preocupante na Amazônia e no Nordeste, onde muitos objetivos e metas não serão alcançados em 20015, e nem nas próximas décadas. Mas o 
que fazer diante da crescente situação adversa de saúde, saneamento e degradação social e ecológica do país?

As ligações entre saúde, qualidade de vida e qualidade do meio ambiente já são bastante conhecidas, a ponto de se tornarem hoje uma obviedade (UNEP, 2003). Os dados nacionais e internacionais ligando queimadas ao aumento de doenças respiratórias e câncer, os dados da Amazônia ligando alguns grandes projetos hidroelétricos e, mais recentemente, o avanço da fronteira da soja ao aumento da malária e da dengue, os efeitos da poluição mercurial na cadeia alimentar das populações ribeirinhas, o efeito da pobreza na degradação ambiental peri-urbana e rural são alguns dos exemplos mais graves das respostas ambientais à influência antrópica (CHIVIAN et al., 1994; COUTO, 2002; GRIFO; ROSENTHAL, 1997; PORTO; FREITAS, 2002; SILVA, 2004a, 2004b). Programas de compensação social, como o Bolsa Família, de saúde pública, como o Programa de Agentes Comunitários de Saúde (PACS) e o Programa de Saúde da Família (PSF), iniciativas como o "SUS Verde" (Projeto Amazônia Legal) (MACHADO, 2003), e mesmos possíveis políticas de geração de emprego e renda que venham a ser implantadas só funcionarão efetivamente para melhorar a qualidade de vida da população se diagnosticarmos claramente os problemas com os quais estamos lidando, se compreendermos a enorme diversidade socioecológica da Amazônia e se integrarmos as populações locais à elaboração e à fiscalização das políticas públicas de saúde e ambiente.

Pessoas doentes e carentes, sem educação e sem expectativa de sobrevivência, isto é, sem Cidadania, são o resultado de um ambiente político e social altamente degradado, e não é possível esperar que em tais circunstâncias o ambiente natural possa ser respeitado ou preservado. A Carta da Terra, aprovada em 2000, em Paris, aponta isso claramente quando clama, em seus quatro princípios centrais, pela indissociabilidade da relação entre as políticas sociais (justiça social e econômica, democracia, não-violência e paz, respeito e cuidado da comunidade de vida) e a qualidade do meio ambiente (integridade ecológica).

No campo propositivo, pelo menos quatro iniciativas são necessárias para promover a melhoria da qualidade de vida e de saúde das populações amazônicas:

a) reforço da vigilância epidemiológica, sanitária e ambiental, com maior participação popular e maior integração entre os múltiplos agentes regionais, para a geração de dados globais e confiáveis sobre as realidades da região; 
Hilton P. Silva

b) aumento dos investimentos em ciência, tecnologia, formação e capacitação continuada de recursos humanos, para o desenvolvimento de infra-estrutura, estratégias e programas baseados nas realidades regionais, voltados para as questões de saúde, meio ambiente e desenvolvimento socioeconômico com bases ambientalmente sustentáveis;

c) aumento da interação, em todas as esferas e níveis de governo, entre as instituições públicas e privadas regionais que lidam com a saúde, com o meio ambiente e com políticas públicas, visando compreender melhor a dinâmica das relações saúdedoença-meio ambiente, em particular no que concerne às doenças emergentes e reemergentes;

d) desenvolvimento de indicadores de saúde e ambiente simples e mensuráveis, que levem em consideração as especificidades microrregionais e a capacidade de participação das comunidades na coleta e na utilização das informações geradas local e regionalmente.

A Organização Panamericana de Saúde estima que, para cada $\mathrm{R} \$$ 1,00 investido em saneamento ambiental, são economizados $R \$ 4,50$ em serviços de saúde. Mas o quanto esse investimento significa em termos de vidas humanas salvas e de preservação dos mananciais, das florestas e da biodiversidade ainda não pode ser calculado. A Amazônia, como um dos muitos "Brasis", é marcada por uma enorme diversidade ambiental, social, econômica e cultural. Dados os inúmeros dilemas impostos pelo gigantismo da região e pela própria "ameaça à soberania nacional", resultantes de descasos históricos e de estratégias geopolíticas recentes, apontados por alguns autores (CABRAL, 1999; CARVALHO, 2004; GASPARINI, 2005; MATTOS, 2005), o principal desafio para a sociedade brasileira no século XXI é decidir como lidar com a megadiversidade que herdamos, em todas as suas múltiplas dimensões. 


\section{REFERÊNCIAS}

ALIER, J. M. Da economia ecológica ao ecologismo popular. Blumenau: Editora da FURB, 1998.

ARRUDA, B. K. G. (Org.). A educação profissional em saúde e a realidade social. Recife: Instituto Materno Infantil de Pernambuco (IMIP), Ministério da Saúde, 2001.

BAKER, P. T.; LITTLE, M. A. Man in the Andes. Stroudsburg: Dowden, Hutchison, and Ross, Inc., 1976.

BENCHIMOL, S. Os índios e os caboclos da Amazônia: uma herança culturalantropológica. Amazônia, Belém, n. 1, p. 98-112, 1998.

BRASIL. Ministério da Saúde. Indicadores e Dados Básicos para a Saúde no Brasil (IDB). Brasília, DF, 2004a.

BRASIL. Ministério da Saúde. Programa Nacional de DST e AIDS. Disponível em: <http://www.aids.gov.br>. Acesso em: 25 out. 2004b.

BRASIL. Ministério da Saúde. DATASUS. Disponível em: <http:// w3.datasus.gov.br/datasus/datasus.php>. Acesso em: 14 mai. 2005.

BRASIL. Senado Federal. Agenda 21: Conferência das Nações Unidas sobre Meio Ambiente e Desenvolvimento. Brasília, DF, 2001.

BRITO, A. M.; CASTILHO, E. A.; SZWARCWALD, C. L. Aids e infecção pelo HIV no Brasil: uma epidemia multifacetada. Revista da Sociedade Brasileira de Medicina Tropical, Uberaba, v. 34, n. 2, p. 207-217, 2000.

CABRAL, B. A problemática da Amazônia Brasileira. Revista do Clube Militar, Rio de Janeiro, n. 363, p. 5-7, 2005.

CARVAlHO, A. D. Amazônia: preocupações, pressões... é hora de novas campanhas. Política e Estratégia Brasileira de Defesa, Segurança e Desenvolvimento, Rio de Janeiro, n. 232, p. 34-36, 2004.

CFM (Conselho Federal de Medicina). Atestado de óbito. Medicina CFM, Brasília, n. 152, p. 22, 2005. 
Hilton P. Silva

CFM (Conselho Federal de Medicina). Os médicos e a saúde no Brasil. Brasília: Conselho Federal de Medicina, 1998.

CHIVIAN, E.; MCCALlY, M.; HU, H.; HAINES, A. (Ed.). Critical Condition: Human Health and the Environment. Cambridge: The MIT Press, 1994.

COIMBRA JR., C. E. A.; SANTOS, R. V.; ESCOBAR, A. L. Epidemiologia e saúde dos povos indígenas no Brasil. Rio de Janeiro: Ed. Fiocruz/Abrasco, 2003.

COSTA, H. L. C.; SANTANA, J. M. C.; MOURA, E. A. F.; FERREIRA, E. A. P.; MAIA, M. L. S. Políticas públicas, desigualdades sociais e crianças no Amazonas. Belém: UNAMAZ, FUA, UFPA, (Série Pobreza e Meio Ambiente na Amazônia, 5).1993.

COUTO, R. C.; CASTRO, E. R.; MARIN, R. A. (Org.). Saúde, trabalho e meio ambiente: políticas públicas na Amazônia. Belém: NAEA, 2002.

CUNHA, M. C.; ALMEIDA, M. B. (Org.). Enciclopédia da Floresta. O Alto Juruá: práticas e conhecimentos das populações. Rio de Janeiro: Companhia das Letras, 2002.

DENEVAN, W. M. Cultivated Landscapes of Native Amazônia and the Andes. Oxford: Oxford University Press, 2002.

DIAS, L. C. S.; FILHO, J. D.; PAES, M. G.; FARIAS, A. N.; AGUIAR, J. C. S. Prevalência de parasitas intestinais em habitantes do rio Negro, Estado do Amazonas, Brasil. Acta Amazônica, Manaus, v. 12, n. 1, p. 65-70, 1982.

DIEGUES, A. C. (Org.). Os saberes tradicionais e a biodiversidade no Brasil. São Paulo: NUPAUB, PROBIO, CNPq, 2000.

DRESSLER, W. W.; SANTOS, J. E. Social and cultural dimensions of hypertension in Brazil: a review. Cadernos de Saúde Pública, Rio de Janeiro, v. 16, n. 2, p. 303-315, 2000.

DUARTE, E. C.; SCHNEIDER, M. C.; PAES-SOUSA, R.; RAMALHO, W. M.; SARDINHA, L. M. V.; JÚNIOR, J. B. S.; CASTILLO-SALGADO, C. Epidemiologia das desigualdades em saúde no Brasil: um estudo exploratório. Brasília: Organização Panamericana de Saúde, 2002.

FAOR (Fórum da Amazônia Oriental). Observatório da cidadania do Pará: políticas públicas e controle popular. Belém, 2003a. 
FAOR (Fórum da Amazônia Oriental). Disponível em: < http://www.faor.org.br/ indsocs.html>. Acesso em: 23 nov. 2003b.

FATHEUER, T.; ARROYO, J. C.; MACHADO, J. A. C. Amazônia: estratégias de desenvolvimento sustentável, uma colaboração para a elaboração de planos de desenvolvimento e Agenda 21. Belém: FASE, DED, FAOR, FETAGRI, NAEA/UFPA, UNIPOP, MPST, CPT, CDG, 1998.

FEIJÃO, A. M. M.; GADELHA, F. V.; BEZERRA, A. A.; OLIVEIRA, A. M.; SILVA, M. S. S.; LIMA, J. W. O. Prevalência de excesso de peso e hipertensão arterial em população urbana de baixa renda. Arquivo Brasileiro de Cardiologia, São Paulo, v. 84, n. 1, p. 29-33, 2005.

FERRARONI, M. J. R.; FILHO, M. M.; FERRARONI, J. J. Parasitas intestinais numa população humana da cidade de Nova Olinda do Norte, Amazonas. Acta Amazonica, Manaus, v. 9, n. 4, p. 657-659, 1979.

FERREIRA, F. H. G.; LANJOUW, P.; NÉRI, M. A new poverty profile for Brazil using PPV, PNAD and Census data. Disponível em: < http:// www.lacea.org/meeting2000/MarceloNeri.PDF>. Acesso em: 25 out. 2005.

FGV (Fundação Getúlio Vargas). Mapa da fome no Brasil. Rio de Janeiro: Fundação Getúlio Vargas, 2001.

GASPARINI, F. O cerco à Amazônia. PensarBrasil, Rio de Janeiro, n. 4, p. 7-14, 2005.

GRIFO, F.; ROSENTHAL, J. (Ed.). Biodiversity and Human Health. Washington, DC: Island Press, 1997.

GUIMARÃES, C. D. Aids no feminino: por que a cada dia mais mulheres contraem AIDS no Brasil? Rio de Janeiro: Editora UFRJ, 2001.

HECKENBERGER, M. J.; KUIKURO, A.; KUIKURO, U. T.; RUSSELL, J. C.; SCHMIDT, M.; FAUSTO, C.; FRANCHETTO, B. Amazonia 1492: Pristine Forest or Cultural Parkland? Science, Washington, v. 301, n. 5640, p. 1710-1714, 2003.

IBGE (Instituto Brasileiro de Geografia e Estatística). Pesquisa de orçamentos familiares 2002-2003: análise da disponibilidade domiciliar de alimentos e do estado nutricional no Brasil. Rio de Janeiro, 2004. 
Hilton P. Silva

IEC (Instituto Evandro Chagas) Trabalho de campo em Saúde Humana e Ambiente nas comunidades da Flona-Caxiuanã, Melgaço-PA. Relatório. Belém, 2000.

JATENE, S. R.; BRITTO, R. C.; MOURA, E. A. F.; SÁ, E. V.; DINIZ, A. A meiavida da criança na Amazônia. Belém: UNAMAZ, UFPA, (Série Pobreza e Meio Ambiente na Amazônia, 3). 1993.

KORMONDY, E. J.; BROWN, D. R. Fundamentos de ecologia humana. São Paulo: Atheneu, 2001.

LISBOA, P. L. B. (Org.). Natureza, homem e manejo de recursos naturais na região de Caxiuanã, Melgaço, Pará. Belém: Museu Paraense Emílio Goeldi/MCT, 2002.

MACHADO, K. O SUS Verde, construindo uma agenda de saúde para a Amazônia Legal. RADIS, Rio de Janeiro, n. 14, p. 8-11, 2003.

MATTOS, C. M. A internacionalização da Amazônia. Revista do Clube Militar, Rio de Janeiro, n. 416, p. 15, 2005.

MONTEIRO, C. A.; BENÍCIO, D. A.; CONDE, W. L.; POPKIN, B. M. Shifting obesity trends in Brazil. European Journal of Clinical Nutrition, Amsterdã, 54, p. 342-346, 2000.

MORÁN, E. F. (Ed.). Developing the Amazon. Bloomington: Indiana University Press, 1981

MORÁN, E. F. (Ed.). The Dilemma of Amazonian Development. Boulder: Westview, 1983.

NUGENT, S. Amazonian Caboclo Society: an essay on invisibility and peasant economy. Oxford: Berg, 1993.

OPAS (Organização Pan-Americana de Saúde). Rede Interagencial de Informações para a Saúde (RIPSA). Indicadores básicos para a saúde no Brasil: conceitos e aplicações. Brasília, 2002.

PORTO, M. F. S.; FREITAS, C. M. (Ed.). Problemas ambientais e vulnerabilidade: abordagens integradoras para o campo da saúde pública. Rio de Janeiro: CESTH/ENSP/FIOCRUZ, 2002. 
RIBEIRO, K. T. S.; MARIN, R. E. A questão ambiental da água e a interface na saúde humana. In: COUTO, R. C.; CASTRO, E. R.; MARIN, R. A. (Org.). Saúde, trabalho e meio ambiente: políticas Públicas na Amazônia. Belém: NAEA/ UFPA, p. 147-178. 2002.

SILVA, H. P. Sócio-ecologia da Saúde e Doença: Os Efeitos da Invisibilidade nas Populações Caboclas da Amazônia. In: Adams, C., Murrieta, R.S.S. G Neves, W.A. (Orgs.) Sociedades Caboclas Amazônicas: Modernidade e Invisibilidade. São Paulo: Annablume/FAPESP, p. 319-345. 2006.

SILVA, H. P. Saúde e doença em relação ao meio ambiente: desafios para as populações em situação de vulnerabilidade. In: IDHS; PNUD; PUC. Coleção Estudos Temáticos sobre os Objetivos de Desenvolvimento do Milênio: Saúde. Belo Horizonte: PNUD, PUC, p. 108-110. 2004a.

SILVA, H. P. Impactos da degradação ambiental na saúde humana: desafios para o século XXI. Sociedade Médica em Revista, Rio de Janeiro, n. 11, p. 8-11, jul.-ago. 2004b. Disponível on-line em: <http://www.somerj.org.br >.

SILVA, H. P. Intestinal parasites as health challenges for amazonian transitional populations: a case study among Caboclo groups. American

Journal of Human Biology, Hoboken, v. 15, n. 2, p. 284 (resumo), 2003.

SILVA, H. P. Aspectos demográficos e médico-epidemiológicos dos residentes na Floresta Nacional da Caxiuanã, Melgaço, Pará. In: LISBOA, P.L.B. (Org.). Caxiuanã: populações tradicionais, meio físico e diversidade biológica. Belém: Museu Paraense Emílio Goeldi/MCT, p. 77-94. 2002 a.

SILVA, H. P. Making the connection: Anthropological and epidemiological considerations about HIV/AIDS pandemic in developing countries. Boletim do Museu Nacional, Nova Série, Antropologia, Rio de Janeiro, n. 62, p. 118, 2002b.

SILVA, H. P. Growth, Development, Nutrition and Health in Caboclo Populations from the Brazilian Amazon. Tese de Doutorado. Columbus: Department of Anthropology, The Ohio State University. 2001.

SILVA, H. P.; JAMES, G. D.; CREWS, D. E. Blood pressure, seasonal body fat, heart rate, and ecological differences in Caboclo populations of the Brazilian Amazon. American Journal of Human Biology, Hoboken, v. 18, n. 1, p.10-22, 2006. 
Hilton P. Silva

SILVA, H. P.; CREWS; D. E.; NEVES, W. A. Blood pressure variation in two rural Amazonian populations from Brazil. American Journal of Human Biology, Hoboken, v. 7, n. 4, p. 535-542, 1995.

SILVA, H. P.; ECKHARDT, R. B. Westernization and blood pressure variation in four Amazonian populations. Collegium Anthropologicum, Zagreb, v. 18, n. 1, p. 81-87, 1994.

STEIN, J. H. (Ed.). Internal Medicine. $4^{\text {th }}$. ed. St. Louis: Mosby, 1994.

UFRGS; UFPA; PUC-Minas; PNUD; IDHS. Coleção Estudos Temáticos sobre os Objetivos de Desenvolvimento do Milênio: Saúde. Belo Horizonte, MG, 2004.

UNEP (United Nations Environmental Programme). GEO Latin American and the Caribbean Environment Outlook, 2003. Mexico, DF, 2003.

WHO. Informe Mundial sobre Água y Saneamiento. Genebra, 2000.

YUSUF, S.; REDDY, S.; ÔUNPU, S.; ANAND, S. Global burden of cardiovascular diseases part I: general considerations, the epidemiologic transition, risk factors, and impact of urbanization. Circulation, Washington, n. 104, p. 2746-2753, 2001. 